\title{
Multi-frequency energy harvesting using thick-film piezoelectric cantilever
}

\begin{abstract}
Due to the fact that the ambient vibration sources are random and unpredictable, the design of energy harvesters responding to multi-frequency is desirable. In this paper, an array lead zirconate titanate (PZT) thick-film cantilevers were designed and fabricated to demonstrate the possibility of harvesting vibration energy from different frequencies. Two configurations of multi-cantilever were fabricated in a form that elevated from the substrate as free-standing structures. One having six cantilevers of constant width but different lengths and another having five cantilevers of constant length but different widths. The experimental results show a magnitude of voltage at around $70 \mathrm{mV}$ in a range of frequency between $220 \mathrm{~Hz}$ to $520 \mathrm{~Hz}$ which is in a good agreement with simulation results.
\end{abstract}

Keyword: Energy harvesting; Thick-film; Free-standing structure; Piezoelectric 